\title{
Acumulação de competências tecnológicas e os processos subjacentes de aprendizagem na indústria metal- mecânica: a experiência da AGCO - Indústria de Colheitadeiras
}

\author{
Pedro Büttenbender*
}

\begin{abstract}
Resumo
Este artigo enfoca 0 relacionamento entre a acumulação de competências tecnológicas e os processos subjacentes de aprendizagem na AGCO (1970-2000), uma empresa fabricante de colheitadeiras agrícolas. A métrica para examinar acumulação de capacidades tecnológicas identifica três funções: processos e organização, produtos e equipamentos. Os processos de aprendizagem são examinados à luz de quatro características: variedade, intensidade, funcionamento e interação, a partir do uso da estrutura de análise existente na literatura. Baseado em estudo de caso individual, este estudo faz uso de evidências empíricas de primeira mão coletadas por meio de múltiplas técnicas e trabalho de campo. Este estudo encontrou que a maneira de acumulação de competências tecnológicas está associada aos diversos processos usados para adquirir conhecimento tecnológico.
\end{abstract}

Palavras-chave: Acumulação de Competências Tecnológicas e Processo de Aprendizagem

\begin{abstract}
This article focuses on the relationship between technological capability accumulation and the learning underlying processes in AGCO (1970-2000) - a producer of harvester machines. The framework for capabilities identifies three technological functions: processes and organization, products and equipment. The learning processes are examined to the light of four characteristic: variety, intensity, functioning and interaction, from the structure existing analysis use in the literature. Based on an individual case-study, this article draws on first-hand qualitative evidence collected through fieldwork. The evidence points to a strong association between the learning processes used in the company and the manner in which the company accumulated its technological capability over time.
\end{abstract}

Key words: Technological Capability-Accumulation Paths and Learning Processes

\section{Introdução}

A partir do início dos anos 1990 os estudos de estratégia corporativa reafirmaram o papel dos recursos internos à empresa - as capacidades tecnológicas - como fonte de inovação e vantagem competitiva (ex. DIERICKX \& COOL, 1989; HAMEL \& PRAHALAD, 1995; TEECE \& PISANO, 1994; PAVITT, 1991; PISANO, 1994). Esses estudos diferenciavam-se das perspectivas convencionais que buscavam explicar a vantagem competitiva de empresa pela sua capacidade de posicionar-se frente a certas forças do ambiente externo (ex. PORTER, 1985). Muitos estudos na década de 1990 enfatizaram a importância do 'conhecimento' para que as empresas criem e mantenham suas capacidades tecnológicas para competir no mercado mundial (ex. LEONARDBARTON, 1998; IANSITI, 1998). No entanto, ainda é necessária a aplicação de modelos analíticos e estudos empíricos que explorem as implicações práticas dos processos de aprendizagem para a acumulação de capacidades tecnológicas em empresas, sobretudo no contexto de economias ou áreas em industrialização.

Por centrar-se em empresas que operam no contexto de industrialização, o foco de análise deste trabalho difere da maioria dos estudos recentes sobre 'conhecimento' e 'capacidades tecnológicas' em empresas industrializadas. Nestas, as capacidades tecnológicas inovadoras já existem. Empresas em economias em

* Professor, pesquisador e pró-reitor da Unijuí - Campos Santa Rosa. E- mail: pedrolb@ unijui.tche.br. Endereço: RS 344, Km 39 - Santa Rosa-RS. CEP $98900-000$ Artigo recebido em novembro de 2004 e aceito para publicação em março de 2005. 
industrialização, no entanto, entram num ramo de negócios com base na tecnologia que adquiriram de outras empresas em outros países. Portanto, em seu estágio inicial, faltam-lhes até as capacidades tecnológicas básicas. Para tornarem-se competitivas e alcançarem as empresas de tecnologia de ponta, elas têm primeiro de adquirir conhecimento para criar e acumular sua própria capacidade tecnológica, engajando-se em um processo de 'aprendizagem' tecnológica.

O termo 'aprendizagem' tecnológica é em geral compreendido em dois sentidos alternativos. O primeiro referese à trajetória ou caminho ao longo do qual segue a acumulação de capacitação tecnológica. A trajetória pode variar ao longo do tempo: a capacitação tecnológica pode ser acumulada em direções e velocidades diferentes. O segundo sentido refere-se aos vários processos pelos quais conhecimento técnico é adquirido pelos indivíduos e convertido para o nível organizacional. Em outras palavras, os processos pelos quais a aprendizagem individual converte-se em aprendizagem organizacional. Neste trabalho, aborda-se a aprendizagem no segundo dos sentidos acima. Daqui em diante, aprendizagem será compreendida como um processo que permite à empresa acumular capacidades tecnológica.

É por meio da acumulação de capacidades tecnológicas que as firmas tornam-se capazes de realizar atividades inovadoras. A competência tecnológica é aqui definida como os recursos necessários para gerar e gerir aperfeiçoamentos incrementais em processos e organização da produção, produtos, equipamentos e projetos de engenharia até o desenvolvimento de novos produtos, processos produtivos ou novas tecnologias que permitem a empresa explorar melhor mercados existentes ou novos mercados. Tais recursos se acumulam e incorporam nos indivíduos (habilidades, conhecimento e experiência) e nos sistemas organizacionais (BELL \& PAVITT, 1995). Baseado em estudo de caso individual, este artigo enfoca as implicações dos processos de aprendizagem para a acumulação de capacidades tecnológicas em nível da empresa. Este relacionamento é examinado na AGCO do Brasil - Indústria de Colheitadeiras, localizada em Santa Rosa durante o período de 1970 a 2000.

A Seção 2 faz uma breve revisão de estudos anteriores. As Seções 3 e 4 apresentam os modelos para examinar a acumulação de capacidades tecnológicas e os processos subjacentes de aprendizagem. O método do estudo subjacente a este artigo é apresentado na Seção 5. A Seções 6 e 7 apresentam as evidências sobre a acumulação de capacidades tecnológicas e os processos de aprendizagem na empresa (1970-2000). A Seção 8 aborda as implicações dos processos de aprendizagem para a acumulação de capacidades tecnológicas e as considerações finais do artigo.

\section{Breve panorama de estudos anteriores}

Os primeiros estudos sobre acumulação de capacidades tecnológicas, que apareceram nos anos 1970, (p. ex. KATZ, 1986; DAHLMAN \& FONSECA, 1978; LALL, 1987; BELL et al., 1982) mostraram não apenas a incidência de atividades inovadoras em empresas em industrialização, mas também a importância de certos mecanismos de aprendizagem para o desenvolvimento de capacidades tecnológicas. Porém, muitos desses estudos limitaram-se a descrever a trajetória de acumulação de capacidades tecnológicas na empresa sem um exame sistemático do papel dos vários processos de aprendizagem na trajetória de acumulação. Ademais, o tratamento dos processos de aprendizagem limitava-se aos processos de aquisição de conhecimento tecnológico de fontes externas à empresa. Os processos de conversão de conhecimento individual em capacidades tecnológicas não eram examinados. Em outras palavras, as implicações dos processos de aprendizagem para a acumulação de capacidades tecnológicas não eram examinados de maneira compreensiva e à luz de uma estrutura analítica adequada.

Durante os anos 1980 verificou-se uma enorme escassez de estudos de acumulação de capacidades tecnológicas e processos de aprendizagem. Somente a partir de meados da década de 1990 é que emergiram novos estudos sobre essas questões. Por exemplo, Kim $(1995,1997$ a) examinou experiências bem sucedidas na indústria automobilística e eletrônica da Coréia do Sul, explorando o papel da liderança na construção do conhecimento através da criação de crises, úteis para a coordenação dos esforços de aprendizagem. Dutrénit (2000) reconstruiu a trajetória de acumulação de capacidades tecnológicas em uma grande empresa de vidro no 
México, enfocando principalmente os problemas encontrados pela empresa para desenvolver uma base de conhecimentos que possibilitasse a construção e a acumulação destas capacidades ao longo dos anos. Movendo-se um passo a frente, Figueiredo (2001) analisou as implicações dos processos subjacentes de aprendizagem para as diferenças entre duas das maiores empresas de aço do Brasil em termos da maneira e velocidade de acumulação de capacidades tecnológicas.

Tais estudos têm fornecido novas explicações sobre o papel das capacidades tecnológicas dos processos de aprendizagem na estratégia de empresas, particularmente naquelas que operam em economias emergentes, como o Brasil. Por isso, é importante o estudo do relacionamento entre questões em tipos de empresa ainda não examinadas na literatura, como é o caso da AGCO - Indústria de Colheitadeiras.

\section{Acumulação de capacidades tecnológicas}

Competência tecnológica é definida como os recursos necessários para gerar e gerenciar a mudança tecnológica. Estes recursos estão incorporados em indivíduos e sistemas organizacionais (BELL \& PAVITT, 1995). A mudança tecnológica em nível da firma é definida como processo contínuo para absorver ou criar conhecimento tecnológico, determinado por fatores externos à firma e pela acumulação de habilidades e conhecimento intrafirma (LALL, 1992). Capacidades tecnológicas foram conceituadas por Bell (1984), Katz (1986), Scott-Kemmis (1988), Lall (1992), Dahlman et al. (1987), Kim (1997), Pack (1987), Enos (1991) e Bell \& Pavitt (1995). Estas conceituações foram discutidas em profundidade nos trabalhos de Dutrénit (2000) e Figueiredo (2001).

\subsection{Estrutura para descrição da acumulação de competência tecnológica}

As capacidades tecnológicas são examinadas aqui à luz da estrutura desenvolvida em Figueiredo (2001), adaptada de Lall (1992) e Bell \& Pavitt (1995). Neste artigo essa estrutura é adaptada para a realidade da indústria metal-mecânica, fabricante de máquinas agrícolas, conforme indicado na Tabela 1. As colunas da estrutura apresentam as capacidades tecnológicas por funções; as linhas, por nível de dificuldade. As funções tecnológicas examinadas são: 'processos e organização da produção', 'produtos' e ‘equipamentos'.

Seguindo Bell \& Pavitt (1995) e Figueiredo (2001) esta estrutura distingue entre capacidades de 'rotina' e 'inovadoras'. As capacidades de rotina são definidas como os recursos para produzir bens e serviços em determinado nível de eficiência, usando-se uma combinação de fatores: habilidades, equipamentos, especificações de produtos e de produção, sistemas e métodos organizacionais. Capacidades inovadoras incorporam recursos adicionais e distintos para gerar e gerir atividades inovadoras. Nesta estrutura, as atividades referentes às diferentes funções tecnológicas são dispostas em sete níveis. As atividades de rotina são dispostas em dois níveis e as atividades inovadoras estão dispostas em quatro níveis. As células da Tabela 1 indicadas com fundo claro representam atividades de rotina; as de fundo escuro os níveis de atividades inovadoras.

\section{Processos subjacentes de aprendizagem}

A aprendizagem é definida como os vários processos pelos quais as habilidades e conhecimentos são adquiridos pelos indivíduos e convertidos, por meio deles, para o nível organizacional (BELL, 1984). Na subseção seguinte é detalhada a estrutura de descrição dos processos de aprendizagem.

\subsection{Estrutura para a descrição dos processos de aprendizagem}

A aprendizagem aqui é definida como sendo os vários processos formais e informais pelos quais indivíduos, e através destes as firmas, adquirem habilidades e conhecimentos técnicos adicionais (BELL, 1984). A aprendizagem também é entendida como um conjunto de processos que permite a empresa acumular capacidades tecnológicas ao longo do tempo (FIGUEIREDO, 2001). Os processos de aprendizagem permitem à empresa acumular as suas próprias capacidades tecnológicas. O desafio de empresas em economias em 
industrialização é administrar a aprendizagem tecnológica, com vistas a alcançar os mesmos patamares de competitividade das empresas em países tecnologicamente avançados (BELL \& PAVITT, 1995; DOSI, 1988; FIGUEIREDO, 2001). A métrica para exame dos processos de aprendizagem é apresentada e detalhada na Tabela 2. 
Tabela 1. Capacidades tecnológicas em empresas em industrialização: Ind. metal mecânica, fabricante de maquinas agrícolas

\begin{tabular}{|c|c|c|c|}
\hline \multirow[b]{2}{*}{$\begin{array}{l}\text { Competências } \\
\text { Tecnológicas }\end{array}$} & \multicolumn{3}{|c|}{ Funções tecnológicas e atividades relacionadas } \\
\hline & $\begin{array}{l}\text { PROCESSOS E ORGANIZAÇão } \\
\text { DA PRODUÇÃo }\end{array}$ & Produtos & EQUIPAMENTOS \\
\hline & \multicolumn{3}{|c|}{ Rotina } \\
\hline $\begin{array}{c}(1) \\
\text { Básico }\end{array}$ & $\begin{array}{l}\text { Coordenação de rotina na planta. Absorção da capacidade da planta. } \\
\text { Planejamento e controle da produção e Controle da qualidade básico. }\end{array}$ & $\begin{array}{l}\text { Fabricação de modelos de máquinas,seguindo especificações } \\
\text { amplamente aceitas (ex: máquinas de operação manual e } \\
\text { mecânica). Controle de Qualidade de rotina. Fornecimento a } \\
\text { mercados nacionais. }\end{array}$ & $\begin{array}{l}\text { Reposição de rotina de componentes e equipamentos. Envolvimento em } \\
\text { instalações e testes de performance de equipamentos }\end{array}$ \\
\hline \multirow[t]{2}{*}{$\begin{array}{c}\text { (2) } \\
\text { Renovado }\end{array}$} & $\begin{array}{l}\text { Coordenação aprimorada de fluxo de produção. Descrição e padronização } \\
\text { de processos. Área de Inspeção e Controle de Qualidade }\end{array}$ & \begin{tabular}{|l} 
Replicação aprimorada de modelos de máquinas, com \\
adaptações. Inspeção e controle de qualidade de rotina. \\
Obtenção de inserção internacional para os seus produtos.
\end{tabular} & $\begin{array}{l}\text { Reposição de equipamentos semi-automáticos (ex: furadeiras múltiplas, } \\
\text { equipamentos de estamparia, tornos semi-automáticos, cabines de } \\
\text { pintura, esteiras de transporte) sob inspeção e controle de qualidade. }\end{array}$ \\
\hline & \multicolumn{3}{|c|}{ Inovadoras } \\
\hline $\begin{array}{c}\text { (3) } \\
\text { Extra-básico }\end{array}$ & $\begin{array}{l}\text { Pequenas e intermitentes adaptações em processos, eliminação de gargalos } \\
\text { no fluxo e alongamento de capacidade produtiva. Introdução de máquinas } \\
\text { semi-automáticas e automáticas, com domínio básico de sua programação. }\end{array}$ & $\begin{array}{l}\text { Replicação e promoção de pequenas adaptações em modelos de } \\
\text { máquinas a serem produzidas. Ajustes as condições dos } \\
\text { mercados (ex: Adaptação de modelos de máquinas da Europa } \\
\text { para o Brasil, 'tropicalização'). }\end{array}$ & $\begin{array}{l}\text { Manufatura e reposição de equipamentos semi-automáticos (ex: } \\
\text { estâmpadeiras e cortadeiras mecânicas, tornos CNC, etc.). Pequenas } \\
\text { adaptações em equipamentos para ajustá-los as condições locais. } \\
\text { Manutenção corretiva ('break-down') de equipamentos convencionais, } \\
\text { semi-automáticos (ex: centros de usinagem, máquinas CNC). }\end{array}$ \\
\hline $\begin{array}{l}\text { (4) } \\
\text { Pré- } \\
\text { intermediário }\end{array}$ & $\begin{array}{l}\text { Alongamentos sistemáticos de capacidade produtiva. Introdução de novas } \\
\text { técnicas organizacionais (5 S's, Controle Estatístico de Produção-CEP, } \\
\text { Círculos de Controle de Qualidade-CCQ, Kanban, Aprimoramento via } \\
\text { Kaisen, JIT, TQC/M). Utilização de Células de Produção. Implantação de } \\
\text { Centros de Usinagem seriada e de precisão. }\end{array}$ & $\begin{array}{l}\text { Aprimoramentos sistemáticos em especificações dadas. } \\
\text { 'Engenharia reversa'. Desenho e desenvolvimento de Modelos } \\
\text { de Máquinas tecnicamente assistidos. Desenvolvimento de } \\
\text { especificações próprias para máquinas agrícolas. }\end{array}$ & $\begin{array}{l}\text { Manufatura, reposição e reforma de equipamentos automáticos (ex: } \\
\text { centros de usinagem, cortadeiras a laser). Engenharia reversa de } \\
\text { componentes e equipamentos e produção própria do ferramental. } \\
\text { Criação de uma estrutura própria de manutenção. Obtenção da } \\
\text { certificação internacional. }\end{array}$ \\
\hline $\begin{array}{c}\text { (5) } \\
\text { Intermediário }\end{array}$ & $\begin{array}{l}\text { Rotinização das inovações organizacionais (5 S's, controle estatístico de } \\
\text { produção-CEP, círculos de controle de qualidade-CCQ, Kanban, } \\
\text { Aprimoramento via Kaisen, JIT, TQC/M).Aprimoramento contínuo de } \\
\text { processos. Integração de sistemas automatizados de processo e PCP (ex: } \\
\text { utilização de robôs e máquinas automáticas). Certificação ISO 9002. Uso } \\
\text { de estação CAM. Aprimoramento de atividades nos centros de usinagem. } \\
\text { Programação de máquinas CNC ao nível operacional. }\end{array}$ & $\begin{array}{l}\text { Aprimoramento contínuo em especificações próprias, com uso } \\
\text { de contribuições/informações dos clientes. Desenho, } \\
\text { desenvolvimento, manufatura e comercialização de produtos } \\
\text { complexos (ex: Uso de estações CAM). Certificação para } \\
\text { desenvolvimento de produto (ex: ISO 9001). }\end{array}$ & $\begin{array}{l}\text { Fabricação própria de ferramentas para produção. Manutenção } \\
\text { preventiva de equipamentos convencionais, semi-automáticas e/ou } \\
\text { automáticas (ex: centros de usinagem, máquinas CNC, etc). Assistência } \\
\text { técnica para fornecedores e outras empresas. Parceria com fornecedores } \\
\text { de equipamentos e prestadores de serviços para manutenção preventiva. }\end{array}$ \\
\hline $\begin{array}{l}\text { (6) } \\
\text { Intermediário } \\
\text { Superior }\end{array}$ & $\begin{array}{l}\text { Uso de estruturas de P\&D, da corporação, para aprimoramento em } \\
\text { processos. Unidades de P\&D e Engenharia em locais geograficamente } \\
\text { diferentes aos da unidade fabril. Integração entre sistemas operacionais e } \\
\text { sistemas corporativos (Ex: Sistemas em rede o o Sistema Supply Chain). } \\
\text { Engajamento em processos de Gestão Ambiental. Preparação para a } \\
\text { Certificação (ex: ISO 14001). }\end{array}$ & $\begin{array}{l}\text { Interação com Centro de P\&D para desenho e desenvolvimento } \\
\text { de produtos complexos, de alto valor agregado, e com } \\
\text { interligação com outras máquinas (ex: produto integrante de } \\
\text { um sistema com interligação, via satélite, com outras etapas da } \\
\text { cadeia agro-produtiva, como: plantio, manejo e gerenciamento } \\
\text { de lavouras). Adoção de componentes com tecnologia } \\
\text { computadorizada. Adição de valor a produtos desenvolvidos } \\
\text { internamente. Engajamento de usuários na definição de } \\
\text { atributos específicos de cada máquina ('customização'). }\end{array}$ & $\begin{array}{l}\text { Manutenção preditiva da planta inteira (ex: desenho e desenvolvimento } \\
\text { dos equipamentos mais adequados à planta). Desenvolvimento de } \\
\text { equipamentos conjuntamente entre o fabricante/fornecedor e o } \\
\text { usuário/cliente. }\end{array}$ \\
\hline $\begin{array}{c}\text { (7) } \\
\text { Avançado }\end{array}$ & $\begin{array}{l}\text { Produção de classe mundial. Desenhos e desenvolvimento de novos } \\
\text { processos baseados na integração entre Engenharia e P\&D. Obtenção de } \\
\text { certificação ISO-14001. }\end{array}$ & $\begin{array}{l}\text { Desenho e desenvolvimento de produtos em classe mundial. } \\
\text { Desenho original via Engenharia e P\&D. }\end{array}$ & $\begin{array}{l}\text { Desenho e manufatura de equipamentos de classe mundial. P\&D para } \\
\text { novos equipamentos e componentes. }\end{array}$ \\
\hline
\end{tabular}

Fonte: Büttenbender (2001), adaptada de Figueiredo (2001) 


\section{Desenho e método de estudo}

A métrica para a descrição dos processos de aprendizagem organiza e classifica os processos de aprendizagem em quatro características e os distribui em quatro níveis diferentes. As características-chave dos processos de aprendizagem são discriminadas como variedade, intensidade, funcionamento e interação. Os níveis dos processos de aprendizagem são organizados em aquisição interna de conhecimentos, aquisição externa de conhecimentos, socialização de conhecimento e codificação de conhecimento. Estes quatro níveis são estudados em dois grupos: O primeiro é formado pelos processos e mecanismos de aquisição de conhecimentos, considerando o contexto individual. O segundo grupo é formado pelos processos e mecanismos de conversão de conhecimentos, considerando o contexto organizacional. Esta estrutura é detalhada na Tabela 2 .

Tabela 2. Métrica para processos de aprendizagem em empresas

\begin{tabular}{|c|c|c|c|c|}
\hline \multirow{3}{*}{$\begin{array}{c}\text { Processos de } \\
\text { Aprendizagem }\end{array}$} & \multicolumn{4}{|c|}{ Características-chaves dos processos de aprendizagem } \\
\hline & Variedade & Intensidade & Funcionamento & Interação \\
\hline & $\begin{array}{c}\text { Ausente - Presente } \\
\text { Limitada-Moderada- } \\
\text { Diversa }\end{array}$ & $\begin{array}{l}\text { Baixa-Intermitente- } \\
\text { Contínuo }\end{array}$ & Ruim-Moderado-Bom & Fraca-Moderada-Forte \\
\hline \multicolumn{5}{|c|}{ Processos e mecanismos de aquisição de conhecimento } \\
\hline $\begin{array}{c}\text { Aquisição } \\
\text { Externa de } \\
\text { Conhecimento }\end{array}$ & $\begin{array}{l}\text { Presença/ausência de } \\
\text { processos para adquirir } \\
\text { conhecimento } \\
\text { localmente ou no } \\
\text { exterior }\end{array}$ & $\begin{array}{l}\text { Modo como a empresa } \\
\text { usa este processo ao } \\
\text { longo do tempo, pode ser } \\
\text { contínuo, intermitente ou } \\
\text { ocorrer uma única vez. }\end{array}$ & $\begin{array}{l}\text { Modo como o processo foi } \\
\text { criado e modo como ele } \\
\text { opera ao longo do tempo. }\end{array}$ & $\begin{array}{l}\text { Modo como um } \\
\text { processo influencia ou- } \\
\text { tro processo de aquisi- } \\
\text { ção externa ou interna } \\
\text { de conhecimento. }\end{array}$ \\
\hline $\begin{array}{c}\text { Aquisição } \\
\text { Interna de } \\
\text { Conhecimento }\end{array}$ & $\begin{array}{l}\text { Presença / ausência de } \\
\text { processos para adquirir } \\
\text { conhecimento fazendo } \\
\text { atividades internas. } \\
\text { Essas atividades podem } \\
\text { ser de rotina ou } \\
\text { inovadoras. }\end{array}$ & $\begin{array}{l}\text { Modo como a empresa } \\
\text { usa diferentes processos } \\
\text { para aquisição interna de } \\
\text { conhecimento. }\end{array}$ & $\begin{array}{l}\text { Modo como o processo foi } \\
\text { criado, e modo como ele } \\
\text { opera ao longo do tempo } \\
\text { tem implicações práticas } \\
\text { para variedade e } \\
\text { intensidade. }\end{array}$ & $\begin{array}{l}\text { Processo de } \\
\text { conhecimento interno } \\
\text { pode ser influenciado } \\
\text { por processo de } \\
\text { aquisição externa. }\end{array}$ \\
\hline \multicolumn{5}{|c|}{ Processos e mecanismos de conversão de conhecimento } \\
\hline $\begin{array}{l}\text { Codifi } \\
\text { Conhe }\end{array}$ & $\begin{array}{l}\text { Presença/ausência de } \\
\text { diferentes processos e } \\
\text { mecanismos para } \\
\text { codificar o } \\
\text { conhecimento tácito. }\end{array}$ & $\begin{array}{l}\text { Modo como processos } \\
\text { como padronização de } \\
\text { operações são } \\
\text { repetidamente feitos. } \\
\text { Codificação ausente e/ou } \\
\text { intermitente pode limitar } \\
\text { a aprendizagem } \\
\text { organizacional. }\end{array}$ & $\begin{array}{l}\text { Modo como a codifica- ção } \\
\text { do conhecimento foi criada } \\
\text { e opera ao longo do tempo. } \\
\text { Tem implicações para o } \\
\text { funcionamento de todo o } \\
\text { processo de conver- são de } \\
\text { conhecimento. }\end{array}$ & $\begin{array}{l}\text { Modo como a } \\
\text { codificação de } \\
\text { conhecimento foi } \\
\text { influenciada por } \\
\text { processos de aqui- } \\
\text { sição do conhecimento } \\
\text { ou por processos de } \\
\text { socialização de } \\
\text { conhecimento. }\end{array}$ \\
\hline $\begin{array}{l}\text { Socialização de } \\
\text { Conhecimento }\end{array}$ & $\begin{array}{l}\text { Presença/ausência de } \\
\text { diferentes processos } \\
\text { através dos quais } \\
\text { indivíduos } \\
\text { compartilham seu } \\
\text { conhecimento tácito. }\end{array}$ & $\begin{array}{l}\text { Modo como processos } \\
\text { prosseguem ao longo dos } \\
\text { anos. Intensidade } \\
\text { contínua do processo de } \\
\text { socialização de } \\
\text { conhecimento pode } \\
\text { influenciar codificação de } \\
\text { conhecimento. }\end{array}$ & $\begin{array}{l}\text { Modo como mecanismos } \\
\text { de socialização do } \\
\text { conhecimento são cria- dos } \\
\text { e operam ao longo do } \\
\text { tempo. Tem implicações } \\
\text { para a variedade e } \\
\text { intensidade do processo de } \\
\text { conver- são de } \\
\text { conhecimento. }\end{array}$ & $\begin{array}{l}\text { Condução de diferentes } \\
\text { conhecimentos tácitos } \\
\text { para um sistema } \\
\text { efetivo. Socialização } \\
\text { pode ser influenciada } \\
\text { por processos de } \\
\text { aquisição externa e } \\
\text { interna de } \\
\text { conhecimento. }\end{array}$ \\
\hline
\end{tabular}

Fonte: Figueiredo (2001) 
Os processos de aprendizagem permitem à empresa criar, desenvolver e manter conhecimento. A estrutura apresentada a Tabela 2 permite descrever, organizar e analisar os processos de aquisição individual de conhecimento e a sua conversão para o nível organizacional.

Este artigo reconhece, de um lado, que a acumulação de capacidades tecnológicas numa empresa pode ser afetada por fatores externos à empresa, como políticas governamentais, macroeconômicas, tecnológicas e industriais (LALL, 1987, 1992; BELL \& PAVITT, 1995; HOBDAY, 1995). De outro, o artigo também reconhece que os processos de aprendizagem podem ser influenciados por características da empresa, por exemplo, o comportamento da liderança e as crenças e normas e cultura da empresa (ARGYRIS E SCHÖN, 1978; SENGE 1990). Porém, os fatores externos e o comportamento da liderança e as crenças e normas e cultura da empresa estão além do escopo da pesquisa.

\section{Método de estudo}

Para examinar 'se' e 'como' ocorreu o desenvolvimento de capacidades tecnológicas na AGCO - Indústria de Colheitadeiras, utilizou-se o método de estudo de caso individual (YIN, 1994). O estudo foi estruturado para examinar as seguintes questões: (i) desenvolvimento da acumulação de capacidades tecnológicas na AGCO Indústria de Colheitadeiras relativas às atividades de desenvolvimento, execução e implantação de projetos industriais para a indústria metal mecânica (1970 a 2000) e (ii) o papel dos processos de aprendizagem no modo e velocidade de acumulação de capacidades tecnológicas na empresa ao longo do tempo. Para examinar essas questões, foi necessária a coleta de evidências, principalmente qualitativas, sobre as atividades tecnológicas e aos vários processos e mecanismos de aprendizagem usados na empresa (1970-2000). Essas evidências foram obtidas através de fontes múltiplas: entrevistas formais com técnicos, engenheiros, gerentes e diretores da empresa (inclusive ex-funcionários); reuniões; análise de documentação (procedimentos, arquivo técnico, dados históricos, etc) e observação direta. A adaptação e validação da estrutura na Tabela 1 foi realizada com base em intensivas interações com especialistas dessa indústria.

\section{Trajetórias de acumulação de capacidades tecnológicas na AGCO - Indústria de Colheitadeiras}

Esta subseção apresenta as trajetórias de acumulação de capacidades tecnológicas da AGCO- Indústria de Colheitadeiras para as funções de organização da produção, produtos e equipamentos, no período de 1970 à 2000, à luz da estrutura apresentada na Tabela 1. A Tabela 3 resume o número de anos que a empresa levou para acumular os diferentes níveis de competências em cada uma das três funções tecnológicas estudadas. As células de fundo escuro representam níveis de atividades inovadoras, enquanto que as demais representam níveis de competência de rotina. A velocidade de acumulação de capacidades tecnológicas foi avaliada pelo número de anos (n) que a empresa necessitou para alcançar níveis específicos de capacidade tecnológica.

O estudo do tempo de cada um dos níveis, conforme detalhado na Tabela 3, sugere que a evolução da acumulação de competência tecnológica para realizar atividades nas três funções tecnológicas ocorreu em diferentes taxas, velocidades e períodos. O inicio da fabricação de colheitadeiras pela AGCO/Santa Rosa ocorreu num período de pouca complexidade tecnológica, também identificada como 'infância industrial' (BELL et al., 1984). $\mathrm{O}$ aprimoramento das capacidades gerou condições para realizar atividades mais complexas, mantendo níveis satisfatórios de competitividade no mercado regional e nacional e, posteriormente, no mercado internacional. As evidências empíricas demonstram que a empresa mais rapidamente acumulou capacidades para desenvolver atividades da função de processos e organização da produção, comparativamente, as atividades das funções de produtos e equipamentos. Por exemplo, a empresa conseguiu acumular competência tecnológica para realizar atividades inovadoras nas três funções tecnológicas estudadas somente a partir da década de 1980. Destaca-se, no entanto, que a empresa necessitou de dez e doze anos, respectivamente nas funções tecnológicas de produtos e de equipamentos, para acumular competência tecnológica para realizar e dominar atividades do nível extrabásico (nível 3). 
Tabela 3. Número de anos de permanência nos níveis de competência tecnológica AGCO/Santa Rosa - período:1970 a 2000

\begin{tabular}{|c|c|c|c|}
\hline \multirow[b]{2}{*}{$\begin{array}{l}\text { Níveis de Competência } \\
\text { Tecnológica }\end{array}$} & \multicolumn{3}{|c|}{ Funções tecnológicas e atividades relacionadas } \\
\hline & $\begin{array}{l}\text { Processos e Organiza- } \\
\text { ção da produção }\end{array}$ & Produtos & Equipamentos \\
\hline (1) Básico & 4 & 7 & 3 \\
\hline (2) Renovado & 3 & 3 & 5 \\
\hline (3) Extra-básico & 7 & 10 & 12 \\
\hline (4) Pré-intermediário & 6 & 4 & 6 \\
\hline (5) Intermediário & 6 & 7 & 4 \\
\hline (6) Intermediário Superior & 4 & 3 & Nível não alcançado \\
\hline (7) Avançado & Nível não alcançado & Nível não alcançado & Nível não alcançado \\
\hline
\end{tabular}

Fonte: Büttenbender (2001).

A empresa começou a operar em condições precárias se comparadas com as atuais referências tecnológicas para as três funções estudadas. O composto tecnológico, disponível na época, acrescido da iniciativa, criatividade e visão empreendedora dos pioneiros gerou, no entanto, as condições necessárias para criar e manter capacidades tecnológicas para a fabricação de colheitadeiras, desde o início da década de 1970. A acumulação de competência tecnológica seguiu o padrão 'produção-investimentos-inovação' (DAHLMAN ET AL., 1987; FIGUEIREDO, 2001).

A acumulação de competência tecnológica resultou da combinação e sequiência de esforços tecnológicos internos e um conjunto de alianças externas. A postura de priorizar produção, investimentos na inovação, sugere indicar que estes investimentos contribuíram na acumulação de competência tecnológica e a alcançar, após trinta anos, elevados índices de competitividade.

No decorrer dos trinta anos, à luz da Tabela 1, a empresa acumulou competência tecnológica de nível intermediário superior (nível 6) para a função de processos e organização da produção e a função de produtos e de nível intermediário (nível 5) para a função de equipamentos. Especificamente, as evidências sugerem que a empresa acumulou competência tecnológica para desenvolver, de forma isolada, algumas atividades dos níveis mais complexos, nas funções de processos e organização da produção e produtos. A empresa conseguiu acumular competência tecnológica para realizar atividades inovadoras nas três funções tecnológicas estudadas somente a partir da década de 1980.

As evidências empíricas sugerem que a empresa, pela significativa interação com o centro mundial de desenvolvimento de colheitadeiras da empresa (Dinamarca), acelerou a velocidade de acumulação de competências, para realizar atividades mais complexas nas três funções tecnológicas estudadas (ARIFFIN \& BELL, 1999). As capacidades acumuladas no decorrer do período estudado demonstram a capacidade da empresa para criar, manter e aprimorar atividades inovadoras e desenvolver atividades mais complexas em cada uma das três funções tecnológicas estudadas, conforme ilustrado na Figura 1. Estas evidências sugerem que a empresa, à luz das contribuições de Figueiredo (2001), está atingindo um estágio de 'maturidade industrial'.

A acumulação de competência tecnológica na função tecnológica de processos e organização da produção foi marcada por vários processos e mecanismos de aquisição e conversão de conhecimento. A evolução também foi influenciada e relacionada com a capacitação tecnológica de máquinas e equipamentos, os sistemas organizacionais e os processos. A evolução foi contínua, conforme ilustrado na Figura 1, e as capacidades tecnológicas foram incorporadas pelos engenheiros, técnicos, operadores e gerentes, e pelos sistemas e estruturas organizacionais, fluxos de conhecimento, e procedimentos de produção, como expresso por Tremblay (1998).

O início da fabricação de colheitadeiras caracterizou-se pelo domínio de atividades do nível de rotina e algumas atividades inovadoras, conforme ilustrado na Figura 1. A empresa necessitou de aproximadamente dez anos para acumular capacidades para superar as atividades de rotina e realizar atividades inovadoras, na função de produtos, o que foi demonstrada pelos aprimoramentos e inovações introduzidas nas colheitadeiras no inicio da 
década de 1980. Estas capacidades foram acumuladas e incorporadas pelos indivíduos (habilidades, conhecimentos explícitos e tácitos) e nos sistemas organizacionais, assim como definidos por Bell \& Pavitt (1995). A empresa estabeleceu diferentes 'links' entre as subsidiarias e a matriz, como sugerido por Ariffin \& Bell (1999) e Hobday (1995), o que contribuiu para aprimorar e fabricar novos modelos de colheitadeiras a partir de 1997/1998.

A acumulação de competência tecnológica na função tecnológica de equipamentos foi marcada por vários processos e mecanismos de aprendizagem, mas a empresa necessitou de aproximadamente vinte anos para conseguir realizar atividades inovadoras (nível extra-básico). As evidências sugerem que a empresa tinha acumulado capacidades para realizar algumas atividades inovadoras, desde o início da fabricação de colheitadeiras (1970), citando, como exemplo, as adaptações e aprimoramentos em equipamentos, desenho e fabricação de ferramental e matrizes.

Figura 1. Comparação gráfica das trajetórias das funções tecnológicas de processos e organização da produção, produtos e equipamentos da AGCO, de 1970 a 2000.

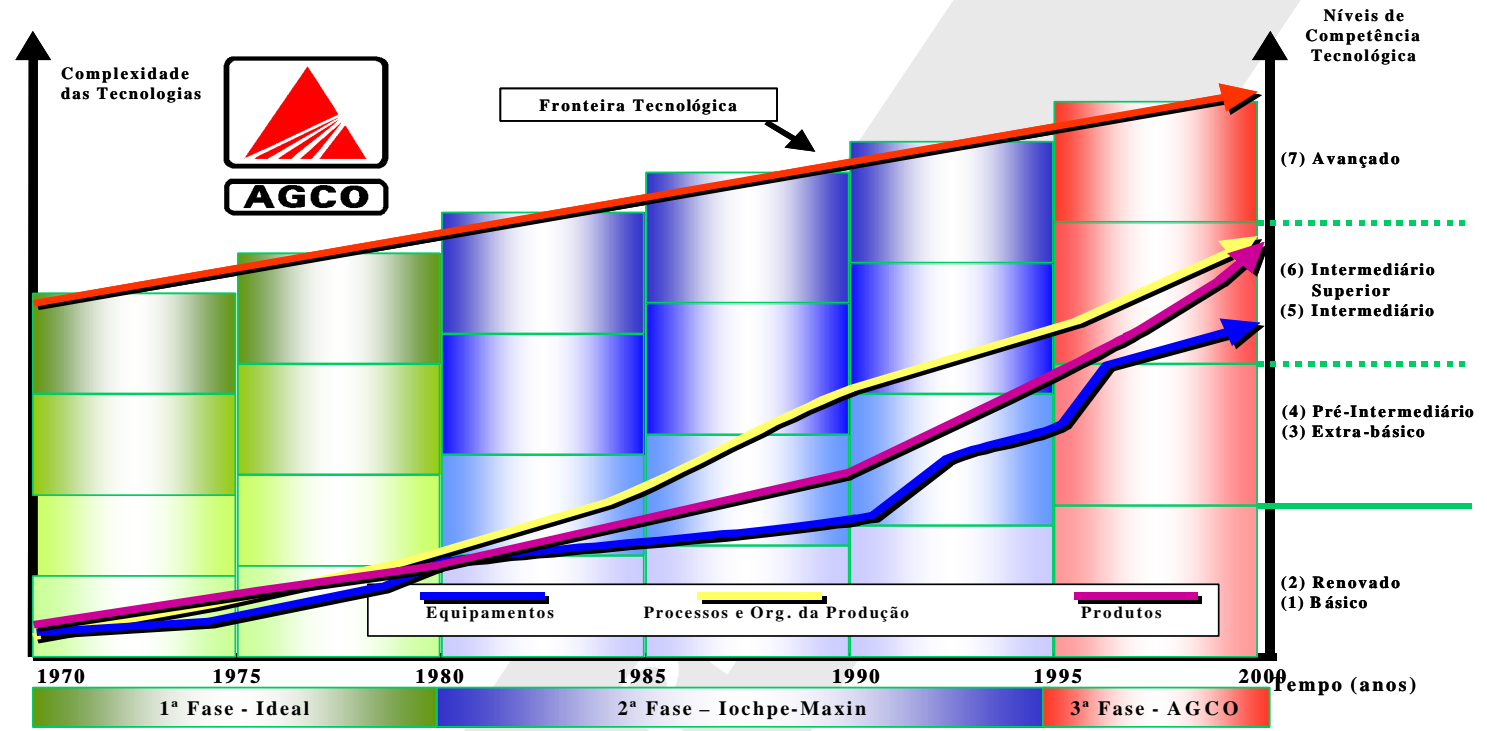

Fonte: Büttenbender (2001).

A velocidade da acumulação de competência tecnológica para realizar atividades na função tecnológica de equipamentos foi menor, comparativamente as demais estudadas, atingindo o nível intermediário (nível 5). Destaca-se que o conhecimento e as capacidades estavam mais concentrados nos indivíduos e, de forma limitada, nos sistemas organizacionais. Estas evidências sugerem uma relação com o estudo de Tremblay (1998) que encontrou limitações quando a competência tecnológica é incorporada em um estoque de indivíduos e não em sistemas organizacionais.

A acumulação de competência tecnológica ao longo do tempo também resultou da capacidade de estimular conflitos e construir consensos, e da incorporação dos recursos para gerar e gerenciar mudanças tecnológicas. As evidências sugerem que a empresa, no período estudado (1970 a 2000), vivenciou as três dimensões/perspectivas apontadas pela literatura de empresas em industrialização (FIGUEIREDO, 2001). As capacidades tecnológicas se acumularam em indivíduos e em sistemas organizacionais, para criar, mudar e aprimorar tecnologia. A acumulação destas capacidades tecnológicas sugere associação aos esforços sistemáticos de aquisição individual de conhecimento e a sua conversão para o nível organizacional. 


\section{Processos de Aprendizagem na AGCO - Industria de Colheitadeiras}

Esta seção analisa as características-chave dos processos de aprendizagem e as suas implicações com as trajetórias de acumulação de capacidades tecnológicas na AGCO - Unidade de Colheitadeiras no período de 1970 à 2000, à luz da métrica na Tabela 2. Os processos de aprendizagem são apresentados e analisados nas três fases da evolução da empresa. As três fases compreendem períodos de tempos diferentes, ou seja, a $1^{\text {a }}$ fase é de 1970 à 1978 (8 anos), a $2^{\text {a }}$ fase de 1978 à 1996 (18 anos) e a $3^{\text {a }}$ fase de 1996 à 2000 (4 anos).

\subsection{Variedade dos processos de aprendizagem}

A variedade foi avaliada em termos de ausência ou presença dos processos de aprendizagem. Os critérios definem a variedade como ausente quando nenhum processo de aprendizagem aconteceu no período em avaliação. ${ }^{1}$ A variedade é definida como presença, quando da existência de processos de aprendizagem no período e, quantitativamente, assim classificados: 'limitado' com um ou dois processos; 'moderado' com três a cinco; ou 'diverso' com seis ou mais. Na Tabela 4 são relacionados os processos de aprendizagem presentes em cada uma das três fases de evolução da empresa. Quantitativamente os processos de aprendizagem são analisados na Tabela 5 .

Os processos de aquisição externa de conhecimento estavam concentrados em poucos mecanismos de aquisição, resultando nos baixos níveis de competência tecnológica, ou seja, níveis de rotina e renovado. O crescimento da variedade dos processos de aquisição de conhecimento resultou em atividades geradoras e difusoras de conhecimentos (LEONARD-BARTON, 1998). Os processos de aprendizagem identificados são apresentados na Tabela 4.

\footnotetext{
${ }^{1}$ Os critérios para o exame das quatro características estão detalhados no estudo subjacente a este artigo (ver BÜTTENBENDER, 2001).
} 
Tabela 4. Processos de aprendizagem da AGCO - Unidade de Colheitadeiras, Santa Rosa/RS, de 1970 à 2000

\begin{tabular}{|c|c|c|c|}
\hline \multirow{2}{*}{$\begin{array}{l}\text { Processos de } \\
\text { Aprendizagem }\end{array}$} & \multicolumn{3}{|c|}{ Processos de Aprendizagem - Três fases da Empresa } \\
\hline & $1^{\mathrm{a}}$ fase -1970 à 1978 & $2^{\mathrm{a}}$ fase -1978 à 1996 & $3^{\mathrm{a}}$ fase -1996 à 2000 \\
\hline \begin{tabular}{|l} 
Processos de \\
Aquisição Externa
\end{tabular} & $\begin{array}{l}\text { - } \quad \text { Contratação de engenheiros e técnicos: } \\
\text { - } \quad \text { Viagens ao exterior: } \\
\text { - } \quad \text { Contratação de "expertise": } \\
\text { - } \quad \text { Contratação de assessorias externas: } \\
\text { - } \quad \text { Presença de técnicos e fiscais do governo }\end{array}$ & $\begin{array}{l}\text { - } \quad \text { Canalização de conhecimento codificado externo: } \\
\text { - } \quad \text { Cursos externos para gerentes, engenheiros e técnicos: } \\
\text { - } \quad \text { Cursos externos para pessoas do nível operacional: } \\
\text { internos: } \\
\text { - } \quad \text { Transferênção de "expertise" para liderar treinamentos } \\
\text { - } \quad \text { Importação de "expertise" para liderar programa de } \\
\text { qualidade: } \\
\text { - } \quad \text { Interação com projeto "parceiros fornecedores": } \\
\text { - } \quad \text { Assessorias externas para gestão de pessoas: } \\
\text { - } \quad \text { Cursos e eventos externos para todos os níveis: } \\
\text { - } \quad \text { Treinamentos no exterior para nível gerencial } \\
\end{array}$ & $\begin{array}{l}\text { - Importação de "expertise" para liderar o sistema } \\
\text { de gestão ambiental; } \\
\text { - Canalização de conhecimento codificado da } \\
\text { Argentina (Deutz-optima); } \\
\text { - Canalização de conhecimento codificado da } \\
\text { Dinamarca; } \\
\text { - Treinamentos no exterior e importação de } \\
\text { especialistas da indústria; } \\
\text { - Cursos externos; e } \\
\text { - Provisão de bolsas de estudo; }\end{array}$ \\
\hline \begin{tabular}{|l} 
Processos de \\
Aquisiçãa Interna
\end{tabular} & $\begin{array}{l}\text { - } \quad \text { Envolvimento em desenho de projeto, projetos de } \\
\text { instalação e operação da rotina da planta; } \\
\text { - } \quad \text { Experiências e esforços de alongamento da capacidade; } \\
\text { - } \quad \text { Aquisição de conhecimento no processo de 'aprender- } \\
\text { fazendo'; e } \\
\text { - } \quad \text { Aprimoramento contínuo nas unidades da planta. }\end{array}$ & $\begin{array}{l}\text { - } \quad \text { Esforços de alongamento da capacidade e manipulação } \\
\text { de parâmetros de processo de produção; } \\
\bullet \quad \text { Aprimoramento contínuo nas unidades da planta e } \\
\text { envolvimento em desenho de projeto; } \\
\bullet \quad \text { Aquisição de conhecimento antes de engajar em novas } \\
\text { atividades; } \\
\bullet \quad \text { Operação de rotina da planta e esforços de alongamento } \\
\text { da capacidade; e } \\
\bullet \quad \text { Cursos internos e aquisição de conhecimento antes de } \\
\text { engajar em novas atividades técnicas. }\end{array}$ & $\begin{array}{l}\text { - } \quad \text { Aquisição de conhecimento antes de engajar em } \\
\text { novas atividades técnicas; e } \\
\text { - Projetos inovadores em parceria com centros de } \\
\text { pesquisa: }\end{array}$ \\
\hline $\begin{array}{l}\text { Processos de } \\
\text { Socialização }\end{array}$ & $\begin{array}{l}\text { - Treinamentos por indivíduos da casa; } \\
\text { - Treinamentos internos ministrados por indivíduos da } \\
\text { casa; e } \\
\text { - } \quad \text { Links para compartilhar conhecimento }\end{array}$ & $\begin{array}{l}\text { - } \quad \text { Formação de grupos e times para solução de problemas; } \\
\text { - } \quad \text { Treinamentos internos por indivíduos da própria } \\
\text { empresa; } \\
\text { - } \quad \text { Solução compartilhada de problemas; e } \\
\text { - } \quad \text { Disseminação de operadores líderes e treinamentos no } \\
\text { trabalho. }\end{array}$ & $\begin{array}{l}\text { - Treinamento interno baseado em programas da } \\
\text { qualidade; } \\
\text { - Treinamentos no trabalho e treinamento de } \\
\text { indivíduos de empresas fornecedoras; } \\
\text { - } \quad \text { Links' para disseminar conhecimento. }\end{array}$ \\
\hline $\begin{array}{l}\text { Processos de } \\
\text { Codificação }\end{array}$ & $\begin{array}{l}\text { - Registro e manipulação de conhecimento próprio; } \\
\text { - Impressão de manuais de fabricação e operação de co- } \\
\text { lheitadeiras; } \\
\text { - Descrição dos procedimentos detalhados de produção; }\end{array}$ & $\begin{array}{l}\text { - Codificação dos projetos de engenharia e manuais de sis- } \\
\text { temas de qualidade; } \\
\text { - } \quad \text { Manipulação de conhecimento codificado próprio; } \\
\text { - Descrição dos padrões dos sistemas de automação; } \\
\text { - Descrição dos conteúdos e conhecimento de treinamento } \\
\text { externo; }\end{array}$ & $\begin{array}{l}\text { - } \quad \text { Registro e publicação dos referenciais estratégicos } \\
\text { da empresa; } \\
\text { - Seminários, eventos internos e intranet; e } \\
\text { - } \quad \text { Registro e acompanhamento individual }\end{array}$ \\
\hline
\end{tabular}

Fonte:Büttenbender (2001) 
A variedade dos processos de aprendizagem sugere a influência positiva dos processos de aprendizagem na definição das trajetórias de acumulação de competência tecnológica da empresa. Estudos desta natureza foram também realizados por Kim (1995 e 1997) que exploraram o papel e influência positiva da liderança corporativa em construir crises e alcançar consensos, contribuindo com os processos de aprendizagem. Os processos de aprendizagem, implementados ao longo do tempo, permitiram acumular as suas próprias capacidades tecnológicas e auxiliaram em dominar recursos necessários para alcançar melhores patamares de competitividade (DOSI, 1988). A empresa carecia, inicialmente, até mesmo de capacidades básicas.

A variedade dos processos de aprendizagem, integrada com as demais características-chave (HOBDAY, 1995; KIM, 1995 e DUTRÉNIT, 2000), gerou as condições e os recursos para criar, manter e aprimorar conhecimento e acumular competência tecnológica para desenvolver atividades mais complexas. A empresa passou a gerir os processos de aprendizagem na dimensão das suas aptidões estratégicas (LEONARDBARTON, 1998). A acumulação de competência tecnológica foi se constituindo ao longo do tempo em vantagem competitiva. Esta competência tecnológica dificilmente poderá ser imitada, pois está integrada no sistema de atividades, nos sistemas físicos, nas bases de qualificações e de conhecimentos, nos sistemas gerenciais de instrução e recompensa, entre outros (LEONARD-BARTON, 1998:35).

Tabela 5. Variedade (n) dos processos de aprendizagem da AGCO do Brasil/Santa Rosa. Período de 1970 a 2000.

\begin{tabular}{cccc}
\hline Processos de & \multicolumn{3}{c}{ Fases e Períodos da Trajetória da Empresa } \\
\cline { 2 - 4 } aprendizagem & $\mathbf{1}^{\mathbf{a}}$ Fase: 1970 a 1978 & $\mathbf{2}^{\mathbf{a}}$ Fase: 1978 a 1996 & $\mathbf{3}^{\mathbf{a}}$ Fase: 1 1996 a 2000 \\
\hline Aquisição externa & $\mathrm{n}=5$ (Moderada) & $\mathrm{n}=10$ (Diversa) & $\mathrm{n}=6$ (Diversa) \\
\hline Aquisição Interna & $\mathrm{n}=4$ (Moderada) & $\mathrm{n}=5$ (Moderada) & $\mathrm{n}=2$ (Limitada) \\
\hline Socialização & $\mathrm{n}=3$ (Moderada) & $\mathrm{n}=4$ (Moderada) & $\mathrm{n}=3$ (Moderada) \\
\hline Codificação & $\mathrm{n}=2$ (Limitada) & $\mathrm{n}=5$ (Moderada) & $\mathrm{n}=3$ (Moderada) \\
\hline Total & $\mathrm{n}=14$ & $\mathrm{n}=24$ & $\mathrm{n}=14$ \\
\hline
\end{tabular}

Fonte: Büttenbender (2001).

\subsection{Intensidade dos processos de aprendizagem}

A intensidade dos processos de aprendizagem, avaliada pela repetibilidade dos processos de aprendizagem ao longo de cada período, foi assim classificada: 'uma vez' quando aconteceu apenas uma vez; 'intermitente' quando aconteceu duas ou três vezes; ou 'contínua' quando aconteceu quatro ou mais vezes. Na Tabela 6 é detalhada a análise da intensidade dos processos de aprendizagem em cada um das fases da empresa.

A intensidade dos processos e aprendizagem caracteriza-se como intermitente na primeira fase e contínua nas duas fases seguintes, demonstradas pela presença significativa desses ao longo do tempo estudado. Os processos de aquisição externa demonstram influência na acumulação de competência tecnológica para desenvolver novas e mais complexas atividades tecnológicas. Os dirigentes, técnicos e engenheiros, participaram de cursos e treinamentos externos, o que contribuiu também na intensidade dos processos aquisição interna, socialização e codificação de conhecimento, incorporando-se a rotina quase diária da empresa, a exemplo do estudo de Gar$\operatorname{vin}(1993)$.

A intensidade dos processos de aprendizagem tem contribuído no entendimento, pelos funcionários, em todos os níveis, da importância e dos princípios envolvidos na tecnologia, a necessidade da inovação e do aprimoramento. Ela tem contribuído também no fluxo de socialização e codificação de conhecimento, qualificando os processos de conversão da aprendizagem individual para a aprendizagem organizacional.

O estudo sugere que os processos de aprendizagem estiveram voltados à criação, manutenção e aprimoramento de conhecimentos. As prioridades voltaram-se para o modo pelo qual a indústria passou a criar e fortalecer as suas próprias competências, a exemplo das contribuições dos estudos de Hobday (1995), Kim (1995) e Dutrénit (2000). Os estudos de Lernard-Barton (1998). 
Tabela 6: Intensidade dos processos de aprendizagem na AGCO do Brasil/Santa Rosa - período de 1970 a 2000

\begin{tabular}{cccc}
\hline Processos de & \multicolumn{3}{c}{ Fases e Períodos da Trajetória da Empresa } \\
\cline { 2 - 4 } aprendizagem & $\mathbf{1}^{\mathbf{a}}$ Fase:1970 a 1978 & $\mathbf{2}^{\mathbf{a}}$ Fase: 1978 a 1996 & $\mathbf{3}^{\mathbf{a}}$ Fase: 1996 a 2000 \\
\hline Aquisição externa & Intermitente & Contínua & Contínua \\
\hline Aquisição Interna & Uma vez & Intermitente & Intermitente \\
\hline Socialização & Intermitente & Contínua & Contínua \\
\hline Codificação & Uma vez & Intermitente & Contínua \\
\hline
\end{tabular}

Fonte: Büttenbender (2001).

\subsection{Funcionamento dos processos de aprendizagem}

O funcionamento foi avaliado em "como" e "modo" pelo qual a empresa organizou e operou os seus processos de aprendizagem ao longo do tempo e as suas contribuições para a variedade e intensidade. A avaliação do funcionamento foi realizada durante as entrevistas, observações diretas e as constatações, considerando as diferenças em relação ao funcionamento dos processos de aprendizagem e as respostas e constatações dos entrevistados (comentários, pontos de vista e avaliações). O funcionamento foi classificado em quatro parâmetros: 'ruim', 'moderado', 'bom', e 'excelente'. A avaliação do funcionamento é detalhada na Tabela 7.

O funcionamento dos processos de aprendizagem oscilou entre ruim, moderado e bom ao longo do tempo estudado. Algumas características do funcionamento dos processos de aprendizagem influenciaram a variedade e a intensidade, como por exemplo: a repetibilidade, a presença ao longo do tempo e interação com os demais processos de aprendizagem influenciaram de forma diferenciada o funcionamento em cada um dos quatro processos, conforme detalhado na Tabela 7.

Tabela 7: Funcionamento dos processos de aprendizagem na AGCO do Brasil/Santa Rosa período de 1970 a 2000

\begin{tabular}{cccc}
\hline Processos de & \multicolumn{3}{c}{ Fases e Períodos da Trajetória da Empresa } \\
\cline { 2 - 4 } aprendizagem & $\mathbf{1}^{\text {a }}$ Fase: $\mathbf{1 9 7 0}$ a 1978 & $\mathbf{2}^{\mathbf{a}}$ Fase: 1978 a 1996 & $\mathbf{3}^{\mathbf{a}}$ Fase: $\mathbf{1 9 9 6}$ a 2000 \\
\hline Aquisição externa & Moderado & Bom & Bom \\
\hline Aquisição Interna & Ruim & Moderado & Moderado \\
\hline Socialização & Ruim & Moderado & Bom \\
\hline Codificação & Ruim & Moderado/Bom & Bom \\
\hline
\end{tabular}

Fonte: Büttenbender (2001).

Inicialmente voltado a resolver problemas técnico-operacionais (1970-1978), as prioridades concentraram-se na aquisição de conhecimento de outras regiões e países, incorporando-o aos processos e organização da produção, produtos e equipamentos. Na segunda e terceira fase, o funcionamento foi bom, considerando, por exemplo, o total de 180.000 de cursos internos e externos à empresa durante o período de 1992 a 2000 . O funcionamento dos processos de aquisição interna de conhecimento foi marcado mais pelo método 'aprenderfazendo' do que pelo método 'aprender-antes-de-fazer'.

\subsection{Interação dos processos de aprendizagem}

A interação foi avaliada pelo modo e como os processos de aprendizagem influenciam um ao outro e como interagiram os processos de aquisição e de conversão de conhecimento. A interação foi classificada nos critérios: 'fraca' quando a influência foi apenas sobre um outro processo de aprendizagem; 'moderada' quando influenciou dois ou três outros processos de aprendizagem; ou 'forte' quando influenciou quatro ou mais processos de aprendizagem. A interação dos processos de aprendizagem é detalhada na Tabela 8. 
Tabela 8. Interação dos processos de aprendizagem. AGCO do Brasil/Santa Rosa - período de 1970 a 2000

\begin{tabular}{lcccc}
\hline Processos de & \multicolumn{4}{c}{ Fases e Períodos da Trajetória da Empresa } \\
\cline { 2 - 5 } aprendizagem & $\mathbf{1}^{\mathbf{a}}$ Fase: $\mathbf{1 9 7 0}$ a 1978 & $\mathbf{2}^{\mathbf{a}}$ Fase: 1978 a 1996 & $\mathbf{3}^{\mathbf{a}}$ Fase: $\mathbf{1 9 9 6}$ a 2000 \\
\hline Aquisição externa & Fraca & Moderada & Forte \\
\hline Aquisição Interna & Fraca & Moderada & Moderada \\
\hline Socialização & Fraca & Moderada & Forte \\
\hline Codificação & Fraca & Moderada & Forte \\
\hline
\end{tabular}

Fonte: Büttenbender (2001).

Apesar de constituir-se em prioridade da empresa, na década de 1970, os processos de aquisição externa apresentaram uma interação fraca, à luz dos critérios de análise. Na segunda fase, e principalmente a partir da década de 1990, os processos de aquisição externa aprimoraram a interação com os demais processos de aprendizagem, em especial, com os processos de socialização e codificação de conhecimento. As evidências descritas para processos de aquisição externa de conhecimento sugerem a interação moderada e forte na segunda e terceira fases respectivamente. Os processos de aquisição de conhecimento, agregados aos demais mecanismos, influenciaram a acumulação de competência tecnológica para o desenvolvimento de atividades mais complexas em cada uma das três funções estudadas.

\section{Conclusões e implicações para gestão}

Este artigo examinou as implicações dos processos subjacentes de aprendizagem para a acumulação de competências tecnológicas. Esse relacionamento foi examinado na AGCO do Brasil - Indústria de Colhetadeiras, localizada em Santa Rosa, RS, durante período 1970-2000. O estudo indica que:

1. Os mecanismos de aquisição e de conversão de conhecimento foram importantes para a acumulação de capacidades na empresa. Isto sugere a importância da interação acumulativa entre os processos de aprendizagem para a construção de competências, defendida por Ariffin \& Bell (1999). As interações entre os processos de aprendizagem, na terceira fase, contribuíram para a emergência de um 'sistema orgânico' de aprendizagem (GARVIN, 1993, LEONARD-BARTON, 1998, TEECE \& PISANO, 1994; FIGUEIREDO, 2001, 2003) - que contribuiu para a realização de atividades inovadoras.

2. As evidências analisadas, à luz dos critérios metodológicos e das contribuições da literatura, sugerem que os processos de aprendizagem, principalmente a partir da década de 1990, contribuíram positivamente para a acumulação de capacidades tecnológicas para realizar atividades mais complexas nas funções de processos e organização da produção, de produtos e de equipamentos. Estas capacidades foram se convertendo em aptidões estratégicas (LEONARD-BARTON, 1998) e que dificilmente poderão ser imitadas.

3. Portanto, alinhando-se a Figueiredo (2001, 2003), as evidências neste estudo indicam que a transição de um nível de acumulação de competência para outro está associada a uma 'descontinuidade' deliberada das características básicas dos processos de aprendizagem. Ou seja, manter inalteradas essas características contribui muito pouco para que a empresa consiga passar, digamos, do nível 3 para o nível 4 e depois para o nível 5. Quanto mais cedo inicia-se a modificação de tais processos, como fez a AGCO, mais rápida será a acumulação de competência tecnológica. O mesmo vale para a manutenção da competência já existente. Os dados deste estudo sugerem que a ACGO não teria conseguido manter a maioria de suas capacidades caso não tivesse reformulado os seus processos de aprendizagem nos anos 1990 .

4. Apesar dos esforços empreendidos, a empresa acumulou, no período de 1970 a 2000, nível intermediário superior (Nível 6) para as funções tecnológicas de 'processos e organização da produção' e 'produtos', o nível intermediário (Nível 5) para a função tecnológica de 'equipamentos'. Isoladamente, a empresa acumulou capacidades tecnológicas para realizar algumas atividades do nível intermediário superior (nível 6) nas funções de 'processos e organização da produção' e 'produtos'. 
5. Adicionalmente, as evidências neste estudo indicam que os processos de aquisição de conhecimento externo e interno são cruciais para as empresas de economias em desenvolvimento, uma vez que elas não podem valer-se de uma base de conhecimentos previamente adquirida. Tanto os processos de aquisição quanto de conversão do saber são complexos e dispendiosos para essas empresas, além de fundamentais para a acumulação de sua capacidade tecnológica. Todavia, ainda que eficazes, somente os primeiros não bastam para acelerar tal acumulação. Sendo assim, a empresa que opera em uma economia ou área em desenvolvimento precisa desenvolver, paralelamente, processos de conversão do saber, tarefa que para ela é ainda mais difícil, complexa e arriscada. Isso porque tais processos não derivam diretamente dos processos de aquisição de saber, e a possibilidade de fracasso aparentemente é maior para os primeiros do que para estes últimos, talvez devido às dificuldades de vencer a resistência interna e garantir-lhes um funcionamento coerente e uma intensidade contínua ao longo do tempo em todos os níveis da empresa. Portanto, a eficácia dos processos de aprendizagem depende de um esforço interno deliberado e cotidiano.

Finalmente, nosso estudo não só alinha-se a estudos que visam explorar o papel cada vez mais significativo da 'aprendizagem' e do desenvolvimento de capacidades tecnológicas na estratégia corporativa, mas também contribui para ampliar o entendimento de como empresas transnacionais que operam no Brasil incorporam essas questões em sua estratégia no intuito de tornarem-se inovadoras em seu mercado. Especificamente, nosso estudo contribui para ampliar o entendimento do processo inovador em um segmento importante da indústria brasileira. Também contribui para expandir nosso conhecimento relativo ao vigor e capacidade criativa de certos segmentos industriais da economia brasileira. Futuros estudos poderão usar as estruturas aqui usadas, para estudos comparativos entre empresas e/ou entre setores industriais, a fim de aprofundar conhecimentos sobre processos de inovação em empresas brasileiras. Em termos de contribuição metodológica este estudo mostra a aplicabilidade das estruturas analíticas para estudar capacidades tecnológicas e processos de aprendizagem (FIGUEIREDO, 2001, 2003) em indústria metal-mecânica, fabricante de máquinas agrícolas. 


\section{Referências bibliográficas}

ARGYRIS, C. \& SCHÖN, D. Organizational Learning: a Theory of Action Perspective, Reading, MA:Addison-Wesley, 1978.

ARIFFIN, N. \& BELL, M. Patterns of subsidiary-parent linkages and technological copability building in Eletronics TNC subsidiaries in Malaysia. In: Jomo, K.S. \& Felker, GH. (eds.). Indsutrial Technology development in Malásia. Routtledge, 1999, pp. 150-90

BELL, M. Learning and the accumulation of industrial technological capacity in developing countries. In: King, K. \& Fransman, M. (eds.). Technological capability in the Third World. London: Macmillan, 1984.

BELL, M., D. SCOTT-KEM MIS \& SATYARAKWIT W. 'Limited Learning in Infant Industry: a Case Study', In F. Stewart and J. James (eds) The Economics of New Technology in Developing Countries. London: Frances Pinter, 1982.

BELL, M. \& PAVITT, Keith. The development of technological capabilities. Technology and International Competitiveness. Washington: The World Bank, 1995.

BELL, M.; ROSS- LARSON, B. \& WESTPHAL, L. E. Assessing the Performance of Infant Industries. World Bank Staff Working Papers n. 666. Washington: The World Bank. 1984.

BÜTTENBENDER, P. L. Acumulação de capacidades tecnológicas e os processos subjacentes de aprendizagem na indústria metal-mecânica: 0 caso da AGCO Comércio e Indústria Ltda, Santa Rosa - RS. Dissertação de Mestrado. FGV-EBAPE, 2001.

DAHLMAN, C. \& FONSECA F. V. From Technological Dependence to Technological Development: The Case of the USIM INAS Steel Plant in Brazil, Working Paper, no 21, IBD/ECLA Research Programme, 1978.

DAHLMAN, C.; ROSS- LARSON, B. \& WESTPHAL, L. Managing Technological Development: Lessons from the Newly Industrializing Countries. World Development, v. 15, n. 6, 1987, pp. 759-775.

DIERICKX, I. \& COOL K. Asset Stock Accumulation and Sustainability of Competitive Advantage. Management Science, v. 35, no 12, 1989, pp.1504-11.

DOSI, G. The nature of the innovative process. In: DOSI, G., FREEMAN, C., NELSON, R., SILVERBERG, G. \& SOETE, L. (eds.). Technical change and economic theory. Londres: Pinter Publishers, 1988.

DUTRÉNIT, G. Learning and Knowledge Management in the Firm. From Knowledge Acumulation to Strategic Capabilities. News Horizons in the Economics of Innovation. Edward Elgar Publishing, 2000.

ENOS, J. L. The Creation of Technological Capability in Developing Countries, London: Printer Publishers, 1991.

FIGUEIREDO, P. N. Technological Learning and Competitive Performance, Cheltenham, UK and Northhampton, MA, USA: Edward Elgar, 2001

. Aprendizagem tecnológica e performance competitiva. Rio de Janeiro: FGV, 2003.

GARVIN, D. A. Building a learning Organisation. Harvard Business Review, v. 71, n. 4, 1993, pp. 78-91, July/August.

HAMEL G. \& PRAHALAD, C. Competindo pelo Futuro. Ed. Campus. Rio de Janeiro, 1995.

HOBDAY, M. Innovation in East Asia: the challenge to Japan. Aldershot, Edward Elgar, 1995.

IANSITT, M. Technology Integration, Boston, MA: Harvard Business School Press. Jacobsson, S. \& Oskarsson (1995), Educational Statistics as an Indicator of Technological Activity'. Research Policy, v. 24, 1998, pp. 127-36.

KATZ, J. Desarrollo y crisis de la capacidad tecnológica latinoamericana. El caso de la Industria Metalmecánica. CEPAL. Buenos Aires, 1986.

KIM, L. Crisis Construction and Organizational Learning: Capability Building in Catching-up at Hyndai Motor. Paper present at the Hitotsubashi-Organization Science Conference, Tokio, October, 1995. $\overline{8} \overline{6}-\overline{100}$

, L. The Dynamics of Samsung's Technological Learning in Semiconductors. California Management Review, v. 39, n 3, 1997, pp.

, L. Imitation to Innovation: The Dynamics of Korea's Technological Learning, Boston, MA: Harvard Business School Press, 1997a.

LALL, S. Learning to industrialise: the acquisition of technological capability by India. London, Macmillan, 1987.

Technological capabilities and industrialization. World Development. London, v. 20, no 2, 1992, pp. 165-186.

LEONARD-BARTON, Dorothy. Nascentes do Saber. Criando e sustentando as fontes de inovação. Rio de Janeiro: Editora Fundação Getúlio Vargas, 1998.

PACK, H. Productivity, Technology and Industrial Development. A Case Study in Textiles, New York: Oxford University Press, 1987.

PAVITT, K. Key Characteristics of the Large Innovating Firm. British Journal of Management, v.2, 1991, pp. 41-50. 
PISANO, G. P. Knowledge Integration, and the Locus of Learning: Na Empirical Analysis of Process Development. Strategic Management Journal, v. 15 (Special Issue), 1994.

PORTER, M. E. Competitive advantage - creating and sustaining superior performance. New York: Free Press, 1985.

SCOTT-KEMMIS, D. 'Learning and the Accumulation of Technological Capacity in Brazilian Pulp and Paper Firms', Working Paper, nํ. 187, World Employment Programme Research (2-22), 1988.

SENGE, P. The Fifth Discipline: The Art and Practice of the Learning Organization. London: Century Business, 1990.

TEECE, D. \& G. PISANO. The Dynamics Capabilities of Firms: an Introduction, Industrial and Corporate Change, v. 3, no 3, 1994 , pp. $537-56$.

TREM BLAY, P.J. Technological capability and productivity growth: an industrialized/ industrializing country comparison. Scientific Series, Montreal, 1998.

YIN, R. K., Case Study Research: Design and Methods, London: Sage. 1994 